\title{
Colistin and anti-Gram-positive bacterial agents against Acinetobacter baumannii
}

\author{
Bin Liu ${ }^{[1],[2], ~ Y o u n i n g ~ L i u}{ }^{[1]}$, Xiuzhen $D i^{[3]}$, Xin Zhang ${ }^{[1]}$, \\ Rui Wang ${ }^{[3]}$, Yan Bai ${ }^{[4]}$ and Jin Wang ${ }^{[3]}$
}

[1]. Department of Respiratory Diseases, Chinese PLA General Hospital, Beijing, China. [2]. Department of Respiratory and Critical Care Medicine, Pingjin Hospital, Logistics University of CAPF, Tianjin, China. [3]. Center of Medicine Clinical Research, Chinese PLA General Hospital, Beijing, China. [4]. Department of Pharmaceutical Care, Chinese PLA General Hospital, Beijing, China.

\begin{abstract}
Introduction: Acinetobacter baumannii has attained an alarming level of resistance to antibacterial drugs. Clinicians are now considering the use of older agents or unorthodox combinations of licensed drugs against multidrug-resistant strains to bridge the current treatment gap. We investigated the in vitro activities of combination treatments that included colistin with vancomycin, norvancomycin or linezolid against multidrug-resistant Acinetobacter baumannii. Methods: The fractional inhibitory concentration index and time-kill assays were used to explore the combined effects of colistin with vancomycin, norvancomycin or linezolid against 40 clinical isolates of multidrug-resistant Acinetobacter baumannii. Transmission electron microscopy was performed to evaluate the interactions in response to the combination of colistin and vancomycin. Results: The minimum inhibitory concentrations (MICs) of vancomycin and norvancomycin for half of the isolates decreased below the susceptibility break point, and the MIC of linezolid for one isolate was decreased to the blood and epithelial lining fluid concentration using the current dosing regimen. When vancomycin or norvancomycin was combined with subinhibitory doses of colistin, the multidrug-resistant Acinetobacter baumannii test samples were eradicated. Transmission electron microscopy revealed that subinhibitory doses of colistin were able to disrupt the outer membrane, facilitating a disruption of the cell wall and leading to cell lysis. Conclusions: Subinhibitory doses of colistin significantly enhanced the antibacterial activity of vancomycin, norvancomycin, and linezolid against multidrug-resistant Acinetobacter baumannii.
\end{abstract}

Keywords: Acinetobacter baumannii. Colistin. Combination. Linezolid. Multidrug-resistant. Vancomycin.

\section{INTRODUCTION}

Over the last decade, Acinetobacter baumannii (A. baumannii) has emerged as one of the most problematic nosocomial pathogens. Successful multidrug-resistant (MDR) clones have been globally disseminated. Colistin is usually considered to be the last treatment option for MDR A. baumannii. However, its efficacy, toxicity and resistance are concerns when used as a monotherapy ${ }^{1}$. The antimicrobial activity of colistin is thought to be mediated by an electrostatic interaction with bacterial lipopolysaccharides, leading to depolarization and disruption of the bacterial outer membrane (OM), and subsequently inducing cell death via osmotic lysis and the generation of toxic hydroxyl radicals. Colistin produces rapid bactericidal effects at high concentrations. At lower concentrations, it affects the

Address: Dr. Youning Liu. Dept. of Respiratory Diseases/Chinese PLA General Hospital. 28 Fuxing Rd., 100853 Beijing, China.

Phone: 8610 6693-7908; Fax: 8610 6693-7166

e-mail: iamicehe@163.com

Received 12 April 2014

Accepted 4 August 2014 bacterial $\mathrm{OM}$ and increases the permeability of Gram-negative bacteria, which facilitates the penetrative ability of other compounds that are usually excluded, such as hydrophobic drugs: rifampicin ${ }^{2}$, macrolides and glycopeptides ${ }^{3}$ (including teicoplanin, telavancin ${ }^{4}$ and daptomycin ${ }^{5}$ ). These compounds not only affect Gram-negative bacteria but also show potent synergistic activity when combined with colistin.

In the current study, we investigated the in vitro activities of the combination treatment of colistin with vancomycin, norvancomycin (named for its special chemical structure, i.e., demethylation at the $\mathrm{N}$-terminus compared to vancomycin) or linezolid against MDR A. baumannii to determine whether these combinations should be considered for clinical use.

\section{METHODS}

\section{Bacterial isolates}

A total of $40 \mathrm{~A}$. baumannii clinical isolates were selected from January 2011 to June 2013 in three general hospitals in the urban area of Beijing, China. The clinical isolates were identified by Vistem (bioMérieux, Marcy-l'Étoile, France). All are isolates of MDR A. baumannii and were confirmed as non-susceptible to $\geq 1$ agent in $\geq 3$ antimicrobial categories (aminoglycosides/antipseudomonal carbapenems/ 
antipseudomonal fluoroquinolones/antipseudomonal penicillins $+\beta$-lactamase inhibitors/extended-spectrum cephalosporins/ folate pathway inhibitors/penicillins $+\beta$-lactamase inhibitors/ polymyxins/tetracyclines) ${ }^{6}$. The initial bacterial inoculum was adjusted to $5 \times 10^{5} \mathrm{CFU} / \mathrm{ml}$. The plate was incubated for $18 \mathrm{~h}$ at $35^{\circ} \mathrm{C}$. An Escherichia coli strain, ATCC 25922, was used as a quality control in each batch of tests.

\section{Antimicrobial agents and minimum inhibitory concentration (MIC) assays}

All powders were obtained from the National Institute for the Control of Pharmaceutical and Biological Products of China (NICPBP, Beijing, China) except colistin (Sigma Chemical Co., St. Louis, MO, USA), tigecycline (Pfizer Inc., PA, USA) and linezolid (Pfizer Inc., NY, USA). Antibiotic stock solutions were prepared according to the manufacturers' recommendations and stored until use.

The MICs were determined for all antibiotics using the broth microdilution method and according to the protocol of the Clinical and Laboratory Standards Institute (CLSI) in 96-well microtiter plates (Costar, Cambridge, Massachusetts, USA) containing freshly prepared Mueller-Hinton II broth (BBL, Becton Dickenson, Sparks, MD). The inoculum was adjusted to approximately $5 \times 10^{5} \mathrm{CFU} / \mathrm{ml}$ in a $100 \mu \mathrm{l}$ final volume, and microtiter plates were visually scored after incubation for $24 \mathrm{~h}$ at $37^{\circ} \mathrm{C}$. The results of the MIC assay were interpreted according to the CLSI breakpoint criteria.

\section{Checkerboard assay}

Antibiotic interactions were evaluated using the checkerboard assay as previously described ${ }^{7}$. A bacterial suspension was prepared using a 1:100 dilution of an overnight culture (approximately $5 \times 10^{5} \mathrm{CFU} / \mathrm{ml}$ of bacteria), and $50 \mu 1$ was inoculated into each well of a 96 -well microtiter plate. Checkerboards were prepared by doubling dilutions of vancomycin, norvancomycin, and linezolid (0 to $512 \mu \mathrm{g} / \mathrm{ml})$ in the horizontal wells and colistin sulfate (0 to $8 \mu \mathrm{g} / \mathrm{ml})$ in the vertical wells. Plates were read after $18 \mathrm{~h}$ of incubation at $37^{\circ} \mathrm{C}$, and the wells without visible signs of growth were identified by placing the plate on a mirrored surface. The Escherichia coli strain ATCC 25922 was used as a quality control. The lowest fractional inhibitory concentration index (FICI) of all nonturbid wells along the turbidity-nonturbidity interface was used to define synergy. The FICI was calculated with the following equation:

$\mathrm{FICI}=\frac{\mathrm{MIC}_{\mathrm{a}} \text { combination }}{\mathrm{MIC}_{\mathrm{a}} \text { alone }}+\frac{\mathrm{MIC}_{\mathrm{b}} \text { combination }}{\mathrm{MIC}_{\mathrm{b}} \text { alone }}$ where ' $\mathrm{a}$ ' represents colistin and ' $b$ ' represents another compound. Synergy and antagonism were defined by FICI values $\leq 0.5$ and $>4.0$, respectively, whereas values from 0.5 to 4.0 were interpreted to indicate no interaction (additivity/indifference) ${ }^{8}$. A breakpoint of $\leq 2 \mu \mathrm{g} / \mathrm{ml}$ was used to determine vancomycin and norvancomycin susceptibility and was consistent with those set for Gram-positive organisms by the Clinical and Laboratory Standards Institute (CLSI) and the European Committee on Antimicrobial Susceptibility Testing (EUCAST) ${ }^{9,10}$.

\section{Time-kill assays}

The killing kinetics of colistin alone and in combination with vancomycin, norvancomycin, and linezolid were assessed for each of the isolates using standard time-kill methodology and viable bacterial counts. Colistin was added at $0.125 \mu \mathrm{g} / \mathrm{ml}$ (1/4 MIC), vancomycin at $16 \mu \mathrm{g} / \mathrm{ml}$, and norvancomycin at $8 \mu \mathrm{g} /$ $\mathrm{ml}$ to mimic the minimal steady-state concentrations achieved with standard dosing regimens of colistin methanesulfonate $(1,000,000 \mathrm{IU} \text { colistin })^{11}$ and continuously infused vancomycin ${ }^{12}$ and norvacomycin ${ }^{13}$. Linezolid was added at $8 \mu \mathrm{g} / \mathrm{ml}$, a concentration that mimics the minimal steady-state plasma concentration achieved with intravenous administration of $600 \mathrm{mg} / 12 \mathrm{~h}^{14}$. Briefly, $20 \mathrm{ml}$ of MHBCA (Mueller-Hinton broth, cation adjusted; Becton Dickinson Microbiology Systems, Cockeysville, MD) was incubated at $37^{\circ} \mathrm{C}$ with the concentrations described above and an inoculum of $5 \times 10^{5} \mathrm{CFU}$ of isolate 21 (A. baumannii-21). Tubes containing $20 \mathrm{ml}$ of medium broth with the inoculum and no antibiotics were used as a control. The bacterial concentration (expressed in $\log _{10} \mathrm{CFU} / \mathrm{ml}$ ) was determined at $0,2,4,8,12$, and 24h. Bactericidal activity was defined as a three-fold $\log _{10}$ reduction in cell numbers compared with the starting inoculum. Synergy was defined as $a \geq 2 \log _{10}$ decrease in bacterial concentration compared with the effect of the most active individual drug ${ }^{15}$. The experiments were repeated three times, and the mean values for the bacteria were compared among the groups for each individual or combination of antibiotic(s). The $\mathrm{CFU} / \mathrm{ml}$ values for the various groups were compared using a one-way analysis of variance (ANOVA); $\mathrm{p}<0.05$ was considered statistically significant.

\section{Transmission electron microscopy}

The $A$. baumannii-21 isolate was subjected to transmission electron microscopy (TEM). The samples were grown overnight in tryptic soy broth (TSB) and then inoculated into flasks containing $0.125 \mu \mathrm{g} / \mathrm{ml}$ colistin, $16 \mu \mathrm{g} / \mathrm{ml}$ vancomycin or a combination of these agents. The samples were then incubated for $4 \mathrm{~h}$ with shaking prior to centrifugation at $10,000 \times \mathrm{g}$ for $10 \mathrm{~min}$ to pellet the cells. The final bacterial count was approximately 1 to $5 \times 10^{8} \mathrm{CFU}$. The supernatant was discarded, and the cells were washed three times with phosphate-buffered saline (PBS) (Sigma, UK) prior to TEM. To fix the cells, $3 \%$ glutaraldehyde in $0.1 \mathrm{M}$ sodium cacodylate was added, and the samples were incubated overnight at $4^{\circ} \mathrm{C}$. To post-fix the cells, $1 \%$ osmium tetroxide was added, and the samples were incubated for $1 \mathrm{~h}$. The samples were then dehydrated in 30, 50, 70, 90 and $100 \%$ graded acetone dilutions with three washes at each stage. Infiltration was achieved with various ratios of propylene oxide and Spurr's resin prior to overnight incubation and polymerization in $100 \%$ Spurr's resin at $70^{\circ} \mathrm{C}$ for $8 \mathrm{~h}$. The sample blocks were trimmed and ultrathinsectioned using a Reichert-Jung Ultracut E Ultramicrotome to produce sections that were approximately $100 \mathrm{~nm}$ thick. These sections were viewed on a Hitachi H-7500 TEM, and images were recorded using a MegaView II CCD camera. 


\section{RESULTS}

All of the isolates of $A$. baumannii were MDR but susceptible to colistin (Table 1). In addition, all of the samples were resistant to vancomycin, norvancomycin or linezolid with MIC values $\geq 256 \mu \mathrm{g} / \mathrm{ml}$ according to the CLSI criteria. However, checkerboard studies analyzed using the FICI method revealed that $21(52.5 \%)$ isolates became susceptible to vancomycin and $17(42.5 \%)$ became susceptible to norvancomycin when these agents were each used in combination with $0.125 \mu \mathrm{g} / \mathrm{ml}$ colistin (1/4 MIC). No antagonisms were observed (Table 2). The combination of colistin and vancomycin showed synergistic effects in $12(30 \%)$ isolates, whereas that of colistin and norvancomycin showed synergistic effects in $3(7.5 \%)$ isolates.
The combination of colistin and linezolid showed synergistic effects in six (30\%) isolates. The MIC of the A. baumannii-21 isolate decreased to $8 \mu \mathrm{g} / \mathrm{ml}$ when linezolid was used in combination with $0.125 \mu \mathrm{g} / \mathrm{ml}$ colistin.

Time-kill curves generated using viable plate counts are shown in Figure 1. Although colistin alone at $0.25 \mu \mathrm{g} /$ $\mathrm{ml}(1 / 2 \mathrm{MIC})$ initially showed bactericidal action against $A$. baumannii-21, rapid bacterial regrowth was observed after $4 \mathrm{~h}$. A combination of vancomycin $(16 \mu \mathrm{g} / \mathrm{ml}$; Figure 1A) or norvancomycin $(8 \mu \mathrm{g} / \mathrm{ml}$ ) with $0.125 \mu \mathrm{g} / \mathrm{ml}$ colistin (only $1 / 4$ MIC) respectively was found to completely eradicate $A$. baumannii-21 within $12 \mathrm{~h}$, and no regrowth was observed even after 48h of incubation (Figure 1B). However, linezolid showed different results when used alone and in combination with colistin, and synergistic effects were observed. The greatest

TABLE 1 - Minimum inhibitory concentration $(\mu \mathrm{g} / \mathrm{ml})$ of 13 antimicrobial agents for 40 isolates of multidrug-resistant Acinetobacter baumannii.

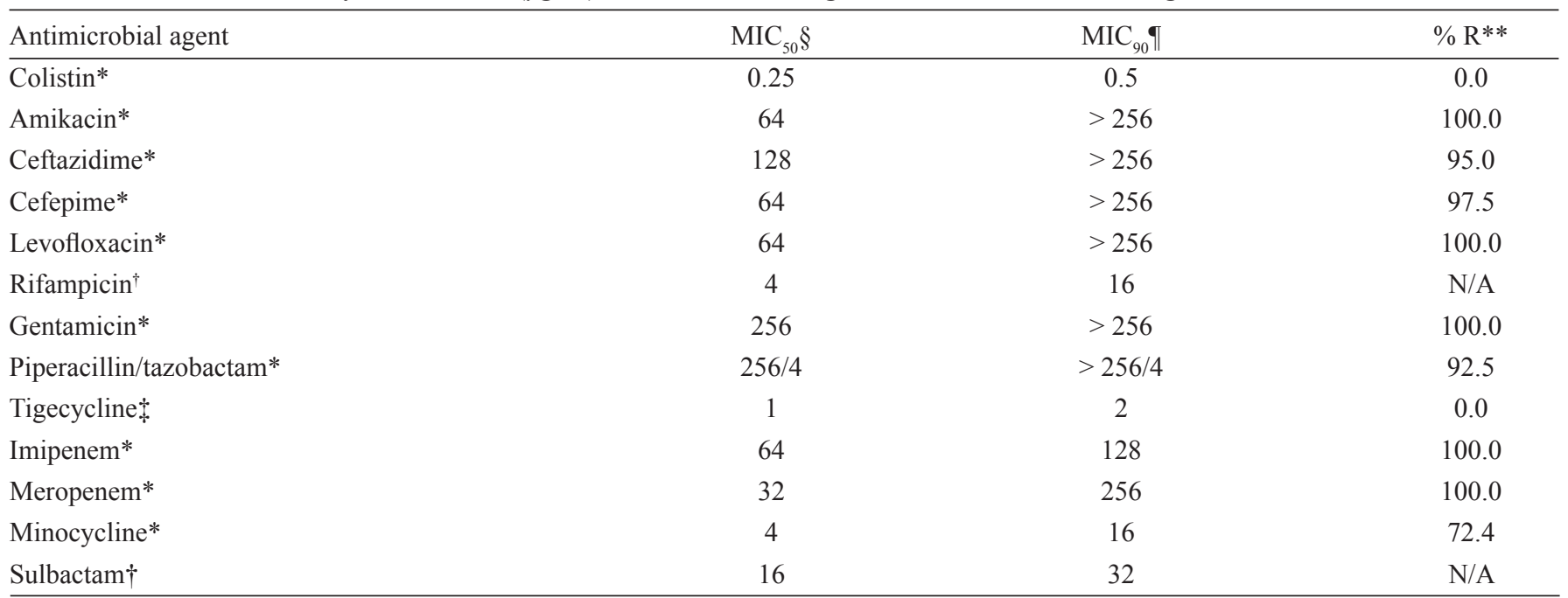

*According to criteria published by the Clinical and Laboratory Standards Institute (CLSI) in 2013; † breakpoint not available (N/A); + United States of America-Food and Drug Administration (USA-FDA) breakpoints were applied when available (Tygacilß package insert, 2012); Tigecycline: susceptible $\leq 2 \mu \mathrm{g} / \mathrm{ml}$, resistant $\geq 8 \mu \mathrm{g} / \mathrm{ml} ; \S \mathbf{M I C}_{50}$ : minimum inhibitory concentrations for $50 \%$ of the organisms; II $\mathbf{M I C}_{\mathbf{9 0}}$ : minimum inhibitory concentrations for $90 \%$ of the organisms. $* * \mathbf{\%} \mathbf{R}$ : percent resistant.

TABLE 2 - Minimum inhibitory concentration $(\mu \mathrm{g} / \mathrm{ml})$ of colistin combined with vancomycin, norvancomycin and linezolid for 40 multidrug-resistant Acinetobacter baumannii clinical isolates.

\begin{tabular}{|c|c|c|c|c|c|c|c|c|}
\hline Agent & \multicolumn{4}{|c|}{ Alone } & \multicolumn{4}{|c|}{ Combination } \\
\hline $\mathrm{CT}$ & $0.125-0.5$ & 0.25 & 0.5 & 100.0 & $0.0625-0.25$ & 0.125 & 0.25 & 100.0 \\
\hline VA & $>256$ & $>256$ & $>256$ & 0.0 & $1-32$ & 2 & 32 & 52.5 \\
\hline NVA & $>256$ & $>256$ & $>256$ & 0.0 & $1-32$ & 4 & 32 & 42.5 \\
\hline
\end{tabular}

CT: colistin; VA: vancomycin; NVA: norvancomycin; LZD: linezolid; $* \mathbf{M I C}_{\mathbf{5 0}}$ : minimum inhibitory concentrations for $50 \%$ of the organisms; $\dagger \mathbf{M I C}_{\mathbf{9 0}}$ : minimum inhibitory concentrations for $90 \%$ of the organisms; $\$ \% \mathrm{~S}$ : percent susceptible. 
(A)
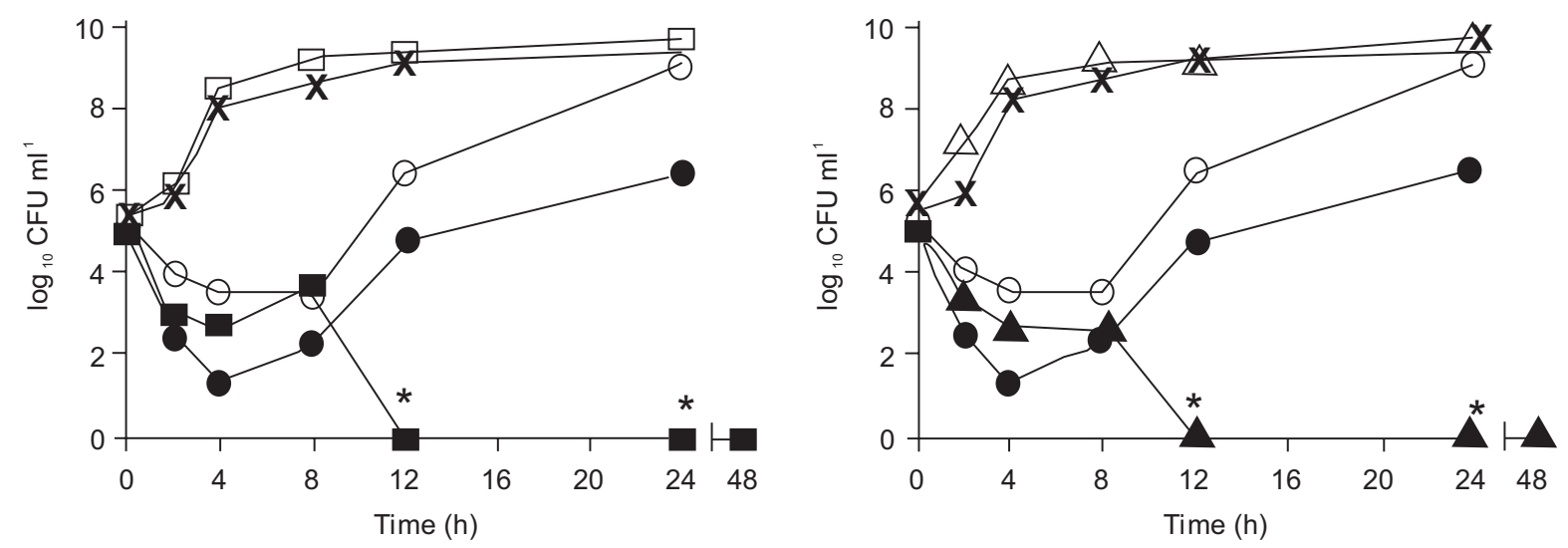

(B)

(C)

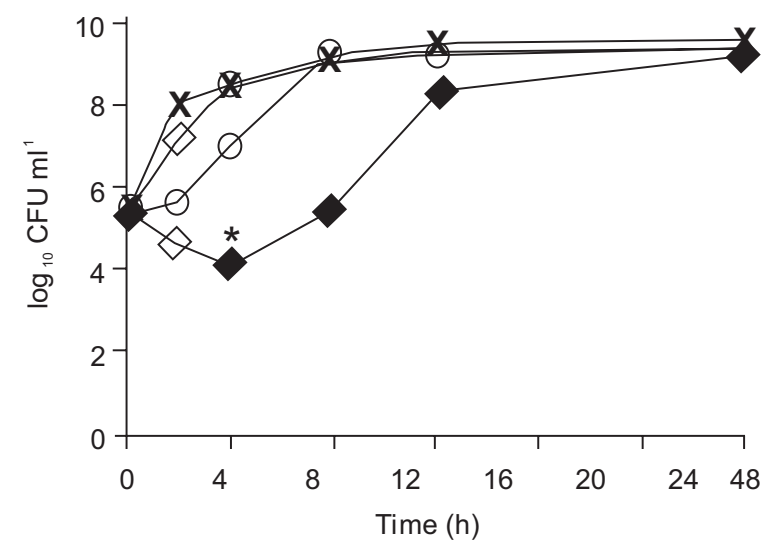

$\mathbf{X}$-Control

$\square$ vancomycin $(16 \mu \mathrm{g} / \mathrm{ml})$

- colistin $(1 / 2 \times M I C, 0.25 \mu \mathrm{g} / \mathrm{ml})$

$\bigcirc$ colistin $(1 / 4 \times \mathrm{MIC}, 0.125 \mu \mathrm{g} / \mathrm{ml})$

$\triangle$ norvancomycin $(8 \mu \mathrm{g} / \mathrm{ml})$

$\diamond$ linezolid $(8 \mu \mathrm{g} / \mathrm{ml})$

$-1 / 4 \times \mathrm{MIC}$ colistin $+16 \mu \mathrm{g} / \mathrm{ml}$ vancomycin

$-1 / 4 \times \mathrm{MIC}$ colistin $+8 \mu \mathrm{g} / \mathrm{ml}$ norvancomycin

1/4xMIC colistin $+8 \mu \mathrm{g} / \mathrm{ml}$ linezolid

FIGURE 1 - Time-kill studies of colistin alone and in combination with vancomycin (A), norvancomycin (B) and linezolid (C). Data were obtained from a clinical isolate of Acinetobacter baumannii (A. baumannii-21). CFU/ml: colony-forming units per milliliter; MIC: minimum inhibitory concentration. ${ }^{*}$ Combination of $1 / 4 \times$ MIC colistin with vancomycin, norvancomycin or linezolid vs. $1 / 4 \times$ MIC colistin $(\mathrm{p}<0.05)$.
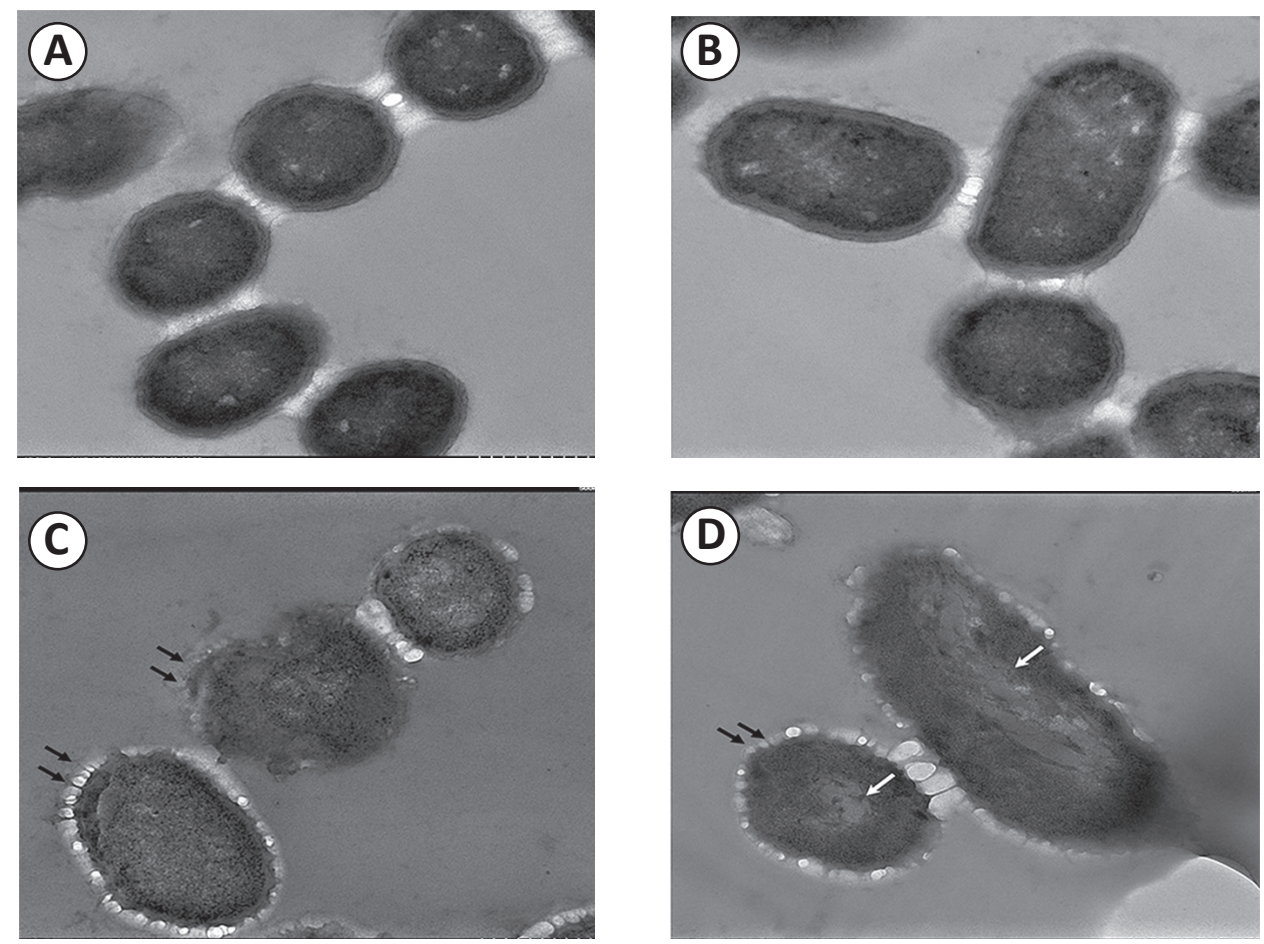

FIGURE 2 - Transmission electron microscopy (TEM) of Acinetobacter baumannii after 4 h of incubation in Mueller-Hinton II broth without drugs (A), with $16 \mu \mathrm{g} / \mathrm{ml}$ vancomycin $(B)$, with $0.125 \mu \mathrm{g} / \mathrm{ml}$ colistin $(C)$, and with $0.125 \mu \mathrm{g} / \mathrm{ml} \mathrm{colistin}+16 \mu \mathrm{g} / \mathrm{ml} \mathrm{vancomycin} \mathrm{(D))}$ TEM Mode: HC-ZOOM; TEM Mag: $12.0 \mathrm{~K} \times$, HV: $80.0 \mathrm{KV}$. 
decrease, which did not exceed $3 \log _{10}$, was achieved at $4 \mathrm{~h}$ (Figure 1C). Bacterial viability was significantly reduced when a combination of drugs was used compared with the that of the control or a single agent $(\mathrm{p}<0.05)$.

Transmission electron microscopy revealed significant differences between cells exposed to colistin/colistin + vancomycin and unexposed controls/vancomycin (Figure 2). Both the control and vancomycin groups were uniform in structure with intact cell walls (Figure 2A and 2B), whereas the colistin and combination groups exhibited markedly diffuse $\mathrm{OM}$ and extensive fibrils (black arrows) that extended from the raised wall bands of MDR A. baumannii (Figure 2C and 2D), indicating an altered OM structure. In addition, ghost cells (white arrows) were commonly observed in the group that received a combination of colistin and vancomycin, indicating subsequent lysis and cell death in A. baumannii.

\section{DISCUSSION}

Considering both the increasing prevalence of MDR Gram-negative bacilli under various hospital settings and the limited number of effective antibacterial drugs against these microorganisms, the identification of an innovative antibiotic strategy, including drugs commonly used only for Gram-positive bacteria, is important. Our research describes the in vitro synergistic activity of colistin in combination with some anti-Gram-positive bacterial agents against MDR A. baumannii. Colistin exhibits good activity against most Gram-negative bacteria, but it is not widely used due to concerns regarding toxicity and tolerability ${ }^{11}$. Several studies have reported poor outcomes in patients with MDR A. baumannii infections treated with colistin, which may be related to the pharmacokinetics and the pharmacodynamics of the drug and to the severity of the underlying illness in the treated patients $^{16}$. The phenomenon of bacterial regrowth observed here may also be a factor that explains the observed lack of a clinical benefit when using this drug.

The OM distinguishes Gram-negative bacteria from Grampositive bacteria and is an effective yet selective permeability barrier ${ }^{17}$. Alterations in the composition and size of porins and/ or in the OM have been shown to alter the sensitivity profiles of bacteria to certain fluoroquinolones, $\beta$-lactam antibiotics, erythromycin and even some of the more recent macrolides.

Glycopeptides, which include vancomycin and norvancomycin and are inhibitors of bacterial peptidoglycan synthesis, damage the peptidoglycan and cause cell lysis due to the turgor pressure of the cytoplasm. Due to their large size and hydrophobicity, glycopeptides are not effective against Gram-negative bacteria. It has been reported that encapsulation in fusogenic liposomes could broaden the spectrum of action of vancomycin against Gram-negative bacteria ${ }^{18}$. The cellpermeabilizing properties of colistin could be exploited to improve the penetration of glycopeptides through the OM of A. baumannii toward their targets in the cell wall ${ }^{19}$. We therefore investigated whether colistin could enhance the activity of vancomycin or norvancomycin or linezolid against $A$. baumannii. An 8- to 256-fold reduction in the MICs of the two drugs was observed in the presence of sub-inhibitory concentrations of colistin. Combination MICs of vancomycin and its structurally related compounds declined below the susceptibility break point in half of the isolates. Synergy was observed (FICI: $\leq 0.5$ ), and the concentrations of the drugs in combination decreased below the minimal steady-state concentrations achieved with standard dosing regimens of colistin ${ }^{11}$ and continuously infused vancomycin $^{12}$ and norvancomycin ${ }^{13}$. The efficacy of low doses of colistin was also supported by the results of the time-kill assays. Here, a $1 / 2 \mathrm{MIC}$ concentration of colistin $(0.25 \mu \mathrm{g} / \mathrm{ml})$ initially showed bactericidal action but did not provide sustained killing over 24h for the tested MDR A. baumannii isolate. In contrast, when a fixed subinhibitory dose of colistin (1/4 MIC) was combined with vancomycin or norvancomycin at clinically relevant serum concentrations, sustained bactericidal activity was maintained for at least $48 \mathrm{~h}$. The risk of nephrotoxicity may be reduced using low-dose colistin $(<0.25 \mu \mathrm{g} / \mathrm{ml})$ with concomitant vancomycin or norvancomycin during the treatment of MDR A. baumannii. The strategy is similar to the use of low-dose aminoglycosides in combination with $\beta$-lactams to treat streptococcal endocarditis ${ }^{20}$. In several anecdotal reports on studies in humans, patients with MDR A. baumannii who received colistin with concomitant vancomycin therapy for associated infections with Gram-positive bacteria did not show any apparent ill effects ${ }^{3}$. Studies in invertebrate animal models, such as the Galleria mellonella model, have indicated that these combinations are highly effective against $A$. baumannii in vivo ${ }^{4}$.

Linezolid, which acts against a broad range of Gram-positive bacteria by preventing the formation of the 70S initiation complex $^{21}$, is likely to be effective for treating respiratory infections ${ }^{14}$ because of its higher concentrations in the epithelial lining fluid (ELF) in response to a twice-daily dosing regimen. It is noteworthy that the MIC of linezolid against $A$. baumannii-21 fell down to the blood and ELF concentration of the drug for the recommended dose regimen $(600 \mathrm{mg} \text { twice daily })^{22}$. A combination of colistin and linezolid could be an option to treat $A$. baumannii pneumonia coinfected with Gram-positive bacteria, especially in patients with renal dysfunction.

To visualize and record the interaction of the combination of colistin and vancomycin with the bacterial cells, TEM analysis was performed on the A. baumannii-21 isolate. The colistin groups showed altered OM structure, reflecting the ability of colistin to disrupt the integrity of bacterial OM. The presence of ghost cells in the samples treated with a combination of colistin and vancomycin indicated the destruction of the cell wall, loss of cytoplasm, and subsequent lysis.

In summary, these data provide primary evidence that subinhibitory doses of colistin significantly enhance the activity of vancomycin, norvancomycin and linezolid against MDR A. baumannii. Subinhibitory doses of colistin in combination with glycopeptides were found to eliminate and prevent the regrowth of MDR A. baumannii for 48h, indicating that these combinations might be useful during nosocomial infections caused by $A$. baumannii. Further work warrants additional in vivo microbiological and pharmacokinetic pre-clinical studies. 


\section{CONFLICT OF INTEREST}

The authors declare that there is no conflict of interest.

\section{FINANCIAL SUPPORT}

This study was supported by the Major National Science and Technology Special Project for New Drugs (Number 2012ZX09303004-002).

\section{REFERENCES}

1. Cai Y, Chai D, Wang R, Liang B, Bai N. Colistin resistance of Acinetobacter baumannii: clinical reports, mechanisms and antimicrobial strategies. J Antimicrob Chemother 2012; 67:1607-1615.

2. Lee HJ, Bergen PJ, Bulitta JB, Tsuji B, Forrest A, Nation RL, et al. Synergistic activity of colistin and rifampin combination against multidrug-resistant Acinetobacter baumannii in an in vitro pharmacokinetic/pharmacodynamic model. Antimicrob Agents Chemother 2013; 57:3738-3745.

3. Gordon NC, Png K, Wareham DW. Potent synergy and sustained bactericidal activity of a vancomycin-colistin combination versus multidrug-resistant strains of Acinetobacter baumannii. Antimicrob Agents Chemother 2010; 54:5316-5322.

4. Hornsey M, Phee L, Longshaw C, Wareham DW. In vivo efficacy of telavancin/colistin combination therapy in a Galleria mellonella model of Acinetobacter baumannii infection. Int J Antimicrob Agents 2013; 41:285-287.

5. Phee L, Hornsey M, Wareham DW. In vitro activity of daptomycin in combination with low-dose colistin against a diverse collection of Gram-negative bacterial pathogens. Eur J Clin Microbiol Infect Dis 2013; 32:1291-1294.

6. Magiorakos AP, Srinivasan A, Carey RB, Carmeli Y, Falagas ME, Giske CG, et al. Multidrug-resistant, extensively drug-resistant and pandrug-resistant bacteria: an international expert proposal for interim standard definitions for acquired resistance. Clin Microbiol Infect 2012; $18: 268-281$

7. Rand KH, Houck HJ, Brown P, Bennett D. Reproducibility of the microdilution checkerboard method for antibiotic synergy. Antimicrob Agents Chemother 1993; 37:613-615.

8. Odds FC. Synergy, antagonism, and what the chequerboard puts between them. J Antimicrob Chemother 2003; 52:1.
9. Clinical and Laboratory Standards Institute (CLSI). Performance standards for antimicrobial susceptibility testing. Twentieth Informational Supplement M100-S23. Wayne, PA, USA: CLSI; 2013.

10. European Committee on Antimicrobial Susceptibility Testing (EUCAST). Breakpoints tables for interpretation of MICs and zone diameters [Software]. EUCAST 2013. [Version 3.0]. Available at: http:// www. eucast.org/clinical breakpoints/.

11. Markou N, Markantonis SL, Dimitrakis E, Panidis D, Boutzouka E, Karatzas $S$, et al. Colistin serum concentrations after intravenous administration in critically ill patients with serious multidrug-resistant, gram-negative bacilli infections: a prospective, open-label, uncontrolled study. Clin Ther 2008; 30:143-151.

12. Pea F, Furlanut M, Negri C, Pavan F, Crapis M, Cristini F, et al. Prospectively validated dosing nomograms for maximizing the pharmacodynamics of vancomycin administered by continuous infusion in critically ill patients. Antimicrob Agents Chemother 2009; 53:1863-1867.

13. Zhang J, Zhang Y, Shi Y, Rui J, Yu J, Cao G, et al. Population pharmacokinetic and pharmacodynamic modeling of norvancomycin. Eur J Clin Microbiol Infect Dis 2008; 27:275-284

14. Conte JE, Golden JA, Kipps J, Zurlinden E. Intrapulmonary Pharmacokinetics of Linezolid. Antimicrobial Agents Chemotherapy 2002; 46:1475-1480.

15. Pillai SK, Moellering Jr RC, Eliopoulos GM. Antimicrobial Combinations, In: Lorian V, editor. Antibiotics in Laboratory Medicine $5^{\text {th }}$ ed. Philadelphia, PA: Lippincott Williams and Wilkins; 2005. p. 365-440.

16. Al-Sweih NA, Al-Hubail MA, Rotimi VO. Emergence of tigecycline and colistin resistance in Acinetobacter species isolated from patients in Kuwait hospitals. J Chemother 2011; 23:13-16.

17. Silhavy TJ, Kahne D, Walker S. The bacterial cell envelope. Cold Spring Harbor Perspect Biol 2010; 2:a000414.

18. Nicolosi D, Scalia M, Nicolosi VM, Pignatello R. Encapsulation in fusogenic liposomes broadens the spectrum of action of vancomycin against Gram-negative bacteria. Int J Antimicrob Agents 2010; 35:553-558.

19. Vaara M. Agents that increase the permeability of the outer membrane. Microbiol Revi 1992; 56:395-411.

20. Hirai Y, Ainoda Y, Shoji T, Totsuka K. Ceftriaxone plus gentamicin or ceftriaxone alone for streptococcal endocarditis in Japanese patients as alternative first-line therapies. J Infect Chemother 2010; 16:186-192.

21. Beibei L, Yun C, Mengli C, Nan B, Xuhong Y, Rui W. Linezolid versus vancomycin for the treatment of gram-positive bacterial infections: meta-analysis of randomised controlled trials. Int J Antimicrob Agents 2010; 35:3-12.

22. Boselli E, Breilh D, Caillault-Sergent A, Djabarouti S, Guillaume C, Xuereb F, et al. Alveolar diffusion and pharmacokinetics of linezolid administered in continuous infusion to critically ill patients with ventilator-associated pneumonia. J Antimicrob Chemother 2012; 67: 1207-1210. 